\title{
Tangence
}

\section{L'historique et le quotidien}

\section{Opération massacre de Rodolfo Walsh : du fait divers à la guerre populaire}

\section{Rita De Grandis}

Numéro 37, septembre 1992

Autopsie du fait divers

URI : https://id.erudit.org/iderudit/025723ar

DOI : https://doi.org/10.7202/025723ar

Aller au sommaire du numéro

Éditeur(s)

Tangence

ISSN

1189-4563 (imprimé)

1710-0305 (numérique)

Découvrir la revue

Citer cet article

De Grandis, R. (1992). L'historique et le quotidien : Opération massacre de Rodolfo Walsh : du fait divers à la guerre populaire. Tangence, (37), 29-44.

https://doi.org/10.7202/025723ar d'utilisation que vous pouvez consulter en ligne.

https://apropos.erudit.org/fr/usagers/politique-dutilisation/ 


\section{L'historique et le quotidien. Opération massacre de Rodolfo Walsh : du fait divers à la guerre populaire}

Rita De Grandis *

Le péronisme est le fait maudit de la politique argentine.

John W. Cooke ${ }^{1}$

Opération massacre fait partie de l'histoire populaire de l'Argentine et relate une des répressions sanglantes accomplie par le régime anti-péroniste. D'abord rumeur, puis fait divers et enfin fait politique, le récit de ce sombre massacre a connu de multiples réactualisations: récits oraux et écrits, articles de revues, films, etc. Le péronisme est, depuis son émergence en 1945, le mouvement nationaliste le plus fort que l'Argentine ait connu. Le fait le plus original et le plus important du péronisme consiste sans doute en la popularité que son fondateur et premier leader, Juan Domingo Perón, a su susciter dans les milieux ouvriers. Perón fut et est encore considéré en Argentine comme une véritable idole, presque un saint. Jean Touchard note à ce propos un de ces couplets entre mille que les Argentins se plaisent à improviser dans leurs manifestations populaires: *Mañana es San Perón; que trabaje el patrón!» («Demain, c'est la Saint-Perón; au travail, le patron $!)^{2}$

Entre *Une famille massacrée à la hache ", le fait divers qui nous est proposé dans ce numéro de revue, et Opération massacre

\footnotetext{
- Simon Fraser University, Departement of Spanish and Latin American Studies. L'auteure tient à remercier Sabine Mabardi et Sylvie Dion pour leur révision de la version française de cet article.

1 En espagnol: * El peronismo es el hecho maldito de la política argentina. " Tiré de l'article de Noé Jitrik, aLas desventuras de la crítica , Las armas y las razones. Ensayos sobre el peronismo, el exilio, la literatura, Buenos Aires, Editorial Sudamericana, 1984, p. 193.

2 Jean Touchard, La République argentine, Paris, Presses Universitaires de France, 1949, p. 56.
} 
(1957) de Rodolfo Walsh, on trouve des points communs et des différences. Les deux récits sont des faits divers relatant un crime. Dans les deux cas, il s'agit d'un massacre, tant dans sa variable nominale (le fait) que dans sa variable verbale (l'action). Dans le premier, il est question du massacre d'une famille par un adolescent et, dans le second, du massacre de pauvres gens par les autorités policières.

Cette «opération massacre * interpelle notre génération, celle des années soixante. Nous avions entendu parler de ce sinistre dépotoir et de l'assassinat de ces ouvriers innocents. Vingt ans après ces événements, les jeunes de cette génération sont devenus les intellectuels des années soixante-dix convertis en masse au péronisme proscrit durant leur enfance (à cette époque, le gouvernement de la "Révolution libératrice" avait été jusqu'à interdire qu'on mentionne le nom de Perón).

\section{Opération massacre: quand le quotidien passe à l'histoire}

L'œuvre journalistique et politique de Rodolfo Walsh compte principalement trois récits: Opération massacre (1957), son récit le plus connu, Le dossier Satanowsky (1958) et Qui a tué Rosendo? (1969) ${ }^{3}$. Cette œuvre peut sembler quantitativement peu importante; elle est cependant remarquable puisqu'elle synthétise à l'extrême l'aspect polémique du discours national de l'époque et qu'elle se situe au carrefour de deux axes distincts, le politique et le littéraire, qui s'opposent et se légitiment à travers le journalisme. La chronique journalistique représente le point de rupture formel le plus important par rapport aux autres formes littéraires con-

3 Rodolfo Walsh, Operación Masacre, Buenos Aires, Ediciones de la Flor, $13^{2}$ édition, 1984. Cet ouvrage, non traduit, est une œuvre inachevée comme nous tenterons de le démontrer dans ce travail. Walsh disparaît le 24 mars 1977 pendant qu'il distribue sa Lettre ouverte à la Junte militaire (Carta abierta a la Junta militar). Un groupe paramilitaire est responsable de son enlèvement. Le Bwenos Aires Herald a été le seul journal à annoncer la nouvelle de sa disparition. La pagination entre parenthèses dans le corps du texte correspond à l'édition citée. Nous traduisons les citations. Caso Satanowsky traite de l'assassinat d'un avocat juif commis par les services secrets argentins. ¿Quién mató a Rosendo? raconte la mort du dirigeant syndical péroniste Rosendo García. Qui a tué Palomino Molero? de Mario Vargas Llosa rappelle le titre du récit de Rodolfo Walsh et recourt au même genre narratif, soit le discours de témoignage. 
temporaines et présuppose une conception de l'œuvre littéraire qui remet en question le caractère autonome de l'esthétiqué Donc, cette ceuvre journalistique s'élabore stratégiquement à partir d'un fait divers, jugé d'emblée comme non esthétique, en réponse à la conception hégémonique d'autonomie du phénomène littéraire. Paradoxalement, ces ouvrages seront lus d'un point de vue littéraire à partir d'une "norme esthétique. (Hans Robert Jauss) qui reconnaît une écriture en dehors des catégories strictement *littéraires 5 . De plus, comme nous le démontrerons, Opération massacre a un statut tout à fait particulier par rapport non seulement au Dossier Satanowsky et à Qui a tué Rosendo?, mais aussi, plus particulièrement, aux revues de gauche des années soixante-dix. En effet, Opération massacre est le point d'origine de toute une série de récits portant sur les assassinats politiques liés au péronisme. Ce traitement des homicides péronistes est, en quelque sorte, étroitement lié à la problématique de la culture et de la littérature nationale. Comme Facundo (1851) de Domingo Faustino Sarmiento et Martín Fierro (1872) de José Hernández, Opération massacre pose la question de la littérarisation des personnages marginaux par rapport à une hégémonie politique et culturelle. L'enjeu, cette fois, est l'inclusion d'une nouvelle figure littéraire, celle de l'ouvrier péroniste.

Opération massacre fait donc le récit d'un événement sanglant. Il raconte l'assassinat, en juin 1956, de gens ordinaires dans un dépotoir de José León Suárez, un quartier de la ville de Buenos Aires. La narrativisation de cet homicide récupère des traits formels caractéristiques du fait divers. L'ouvrage de Rodolfo Walsh (1927-1976) fut d'abord un pamphlet, une "feuille volante ", apparue en marge du journalisme institutionnel dans *un sous-sol de la rue Leandro Alem, ou se publie l'information syndicale. (p. 13). Ce matériel a subi de nombreuses mutations. D'abord publié sous la forme de résultats d'enquête publique dans des revues périodiques à caractère politique, Opération massacre deviendra peu à peu un livre qui sera édité à trois reprises en treize ans, entre 1956 et $1969^{6}$. En se distinguant de l'écriture

4 Pour cet aspect du caractère autonome de l'esthétique, nous renvoyons le lecteur à l'étude d'Antonio Gómez-Moriana, - Bajtin y Adorno frente a la autonomía (relativa) de lo estético, de b literario , manuscrit inédit, 1988.

5 Voir Rita De Grandis, "Le rapport à l'institution. Un exemple argentin. Stratégies de fictionalisation *, à paraître.

6. Ibid. 
journalistique, Opération massacre s'est inséré dans la littérature argentine pour devenir un texte conflictuel et séduisant à la fois.

Au début, les textes de Walsh n'ont pas été retenus par la critique, particulièrement par la critique académique, à cause de leur manque de valeur esthétique et de leur caractère fortement dénotatif (documentaire). Tout le travail critique sur Walsh a dû en même temps se légitimer et légitimer l'inclusion de son ouvre dans l'histoire littéraire. À ce jour, la critique universitaire en Argentine ne l'a pas encore entièrement reconnue, en raison de l'image de Walsh comme auteur à caractère fortement politique et journalistique, ponctuel et historique. Par contre, ce n'est plus le cas hors de l'Argentine; Walsh est maintenant reconnu ailleurs, surtout depuis les années soixante-dix avec l'émergence dans les lettres latino-américaines du discours de témoignage.

Pratique jusqu'alors inhabituelle en littérature, Opération massacre reprend une caractéristique du fait divers: la répétition, la multiplication du récit, son insertion dans une "série noire"? En cela, Walsh révèle une constante de la littérature argentine en ce qui concerne le traitement de la mort à caractère politique. Cette image de la mort fait partie de la mémoire collective du peuple argentin et de son imaginaire politique. Presque vingt ans plus tard, Opération massacre servira de modèle à la presse de la gauche péroniste.

Bien que la critique rende compte du fait que les récits de Walsh se nourrissent des nouvelles qui apparaissent dans la chronique des journaux, nous n'en sommes pas si sûrs ${ }^{8}$. En outre, nous avons constaté que la presse officielle de l'époque ne fait pas mention de cette Opération massacre; elle parle de "coups de feu ", d'* incidents avec des manifestants ", de " raids ", de mots d'éloges «au chef de la police qui a su défendre "la Révolution libératrice" ", etc., mais pas d'assassinat d'ouvriers 9 . Le récit

7 Voir à ce sujet Michel Sailhan, séries noires *, Faits divers, Autrement, $n^{\circ} 98$, avril 1988 , p. $69-87$.

8 Angel Rama souligne que les événements sur lesquels Walsh base ses récits sont empruntés à la page des fait divers dans les journaux. Voir - La narrativa en el conflicto de las culturas", Argentina Hoy (Alain Rouquié, comp.), Mexico, 1982, p. 249-279.

9 Nous avons vu dans La prensa et la nación de la semaine du 10 juin 1956 que divers articles se réfèrent à l'événement du coup raté de valle. Mais aucune allusion aux ouvriers du dépotoir. 
de ce massacre a donc d'abord paru dans les journaux non officiels ${ }^{10}$.

L'assassinat raconté par le journaliste Rodolfo Walsh reprend les caractéristiques structurales du fait divers, mais révèle également le penchant de l'auteur pour le genre policier et la politique. En effet, loin d'être uniquement le récit immanent d'un meurtre qui, selon Roland Barthes, ne renverrait qu'à lui-même, sans référence à d'autres faits ${ }^{11}$, Opération massacre, tout en étant un véritable fait divers, ne peut se dissocier d'une connaissance extérieure à l'événement, c'est-à-dire du climat politique d'alors et de son lien avec l'exécution de nombreux travailleurs. Ces derniers étaient tous reliés de près ou de loin au régime pé: oniste, mouvement politique qui avait été détrôné par un gouvernement militaire, "Révolution libératrice ". Apparemment, ces travailleurs étaient impliqués dans une conspiration, organisée à l'intérieur même de l'armée par les généraux péronistes Valle et Tanco, contre les militaires qui avaient pris le pouvoir au moyen d'un coup d'état. Ce contre-coup devait avoir lieu dans la nuit du 6 juin 1956. À la suite de l'échec de ce projet, le général Valle sera exécuté à son tour le 12 juin 1956. En somme, même si l'on découvre la nature politique de cet assassinat (ce qui est le but visé par le journaliste) il reste néanmoins que cet événement demeure un fait divers qui nous renvoie à la nature populaire et conflictuelle du péronisme en tant que mouvement de masse.

Le récit de Walsh se divise en trois parties: "Les protagonistes ", "Les faits " et "Le procès *. Le récit commence par "Les protagonistes . Comme dans les faits divers, les dramatis personae sont caractérisées par leurs sentiments, leurs émotions. Le narrateur mentionne, par exemple, que *Nicolás Carranza n'était pas un homme heureux cette nuit-là, du 9 juin 1956" (p. 23). Ils appartiennent à une classe sociale spécifique, la classe ouvrière. Le narrateur introduit chacun des protagonistes par une description de son quartier et de sa famille. On apprend que le quartier Florida, où les événements se sont déroulés, est un quartier de

10 Opération massacre, avant la première édition, a paru dans trois hebdomadaires: Propósitos, qui publie la dénonciation de Livraga, le 23 décembre 1956; Revolución nacional et Mayoria, qui publient entre le 27 mai et le 29 juillet 1957 neuf articles brefs de Walsh.

11 Roland Barthes, "Structure du fait divers *, Essais critiques, Paris, Seuil, 1966. 
grands contrastes. À Florida, on trouve le pauvre et le riche, un chalet d'été à côté d'un terrain inculte rempli de mauvaises herbes (p. 31). Les maisons des protagonistes sont des maisons d'ouvriers, propres et sobrement meublées. La décoration inclut des éléments de la culture populaire, une photo du chanteur de tango Carlos Gardel par exemple. Les portraits de famille indiquent qu'ils font partie de bonnes et dignes familles d'ouvriers, où les rôles sociaux ne sont pas remis en cause. Ils sont issus de familles nombreuses et eux-mêmes ont beaucoup d'enfants. Le père est le modèle du chef de famille. Donc, ces ouvriers, les morts comme les survivants, n'ont rien de bien héroïque. Ce sont des gens ordinaires, condamnés par la fatalité et dont la seule gloire se réduit à faire l'objet d'un fait divers. Pour le narrateur, il est clair que ce sont des victimes innocentes. Giunta, un des survivants et témoin du drame, en est l'exemple le plus évident.

La deuxième partie, "Les faits", relate chronologiquement les événements, de l'arrestation jusqu'à l'assassinat, puis les péripéties judiciaires des survivants jusqu'à la libération de deux d'entre eux, Giunta et Livraga. Voici un résumé des événements. Un groupe de 12 à 14 travailleurs (le nombre restera approximatif), rassemblés chez Horace, sont surpris par des policiers vêtus d'uniformes de l'armée argentine qui font irruption dans la maison. Le chef du groupe des policiers demande alors aux personnes présentes: •Où est Tanco?* (c'est le général rebelle). Cette question est aussi le titre du premier chapitre de cette partie. Ce policier, nous l'apprendrons plus tard, n'est autre que le chef de la police de la province de Buenos Aires, le lieutenant-colonel retraité Desiderio A. Fernández Suárez.

Dans un premier temps, les prisonniers sont embarqués dans un autobus urbain réquisitionné $\mathbf{4 5}$ minutes avant l'arrestation au bureau de police du quartier San Martín, dans la rue " 9 de Julio". Après quelques heures, le chef de police donne l'ordre de les conduire dans un terrain vague et de les fusiller. Ils sont alors transportés dans un camion hermétiquement clos, de sorte qu'il leur sera impossible d'identifier l'endroit où on les emmène. Ce lieu était en fait le dépotoir de José León Suárez. Arrivés à destination, les policiers déchargent leurs fusils sur les prisonniers. Cependant, tous ne seront pas tués; il y aura cinq morts et sept survivants dont un blessé. Ces événements ont eu lieu entre le 11 et le 12 juin, en même temps que la promulgation par le gouver- 
nement de la * Révolution libératrice * de la loi martiale abolissant toute garantie constitutionnelle.

La troisième partie, *Le procès ", traite des poursuites judiciaires intentées par les survivants après l'assassinat, plus particulièrement du dossier Livraga ${ }^{12}$. Le journaliste décide d'interviewer Livraga, le survivant, et de reconstituer les faits à l'aide du dossier judiciaire que Livraga produit contre les autorités gouvernementales. Donc, le journaliste Walsh raconte minutieusement ce qui est survenu la nuit du 11 juin: crime commis contre un péroniste, circonstances du drame, la rébellion des généraux Valle et Tanco. Le récit de cet événement nous présente trois points de vue: celui des témoins survivants, celui de la rumeur publique qui a suivi les événements, celui du journaliste.

On assiste à un jeu d'inversion entre la vie et la mort. Le point de départ du récit est la survie des protagonistes, particulièrement celle de 'Troxler et de Livraga. Ces derniers n'hésitent pas à fournir au journaliste des renseignements sur ce qu'ils ont vu. L'énonciateur exalte surtout l'attitude héroïque des victimes du massacre. Le syntagme "Un fusillé qui vit " sera la figure de rhétorique (oxymore) montrant la rupture logique du récit. En effet, la narration évolue autour de cette figure symbolique d'un mort qui parle. Ce mort, Livraga, semble avoir été choisi par le journaliste pour dénoncer l'omission volontaire de la presse officielle, cette dernière ayant refusé de rapporter les déclarations dénonciatrices de la victime. Le journaliste remplit ce vide laissé par la presse officielle, en publiant le déroulement de la poursuite judiciaire intentée par Livraga contre le policier Fernández Suárez, responsable de l'arrestation. Nous retrouvons ici un autre trait caractéristique du fait divers, lequel, selon Roland Barthes, rapproche deux termes qualitativement distants: le fait que le chef de police Fernández Suárez, responsable de l'arrestation, est aussi le meurtrier. Les preuves recueillies après des mois d'enquête permettent au journaliste de conclure à la culpabilité de celui-ci. En effet, le policier est le criminel. Le crime a un effet boomerang; le policier (représentant du pouvoir) en charge d'élucider les causes de l'homicide devient lui-même le responsable direct de la fusillade du dépotoir.

12 Cette partie subira beaucoup de changements dans les trois éditions. Voir Rita De Grandis, op. cit. 
36

Finalement, Opération massacre nous propose aussi un autre point de vue sur la dualité vie-mort, celui du journaliste luimême. Paradoxalement, Rodolfo Walsh, vingt ans après, subit le sort qui aurait dû être celui de son personnage Livraga, quand il devient lui-même la victime d'une autre répression anti-péroniste. Alors que le protagoniste Livraga aurait dû mourir en 1956, le comble de l'ironie, pour un auteur qui se sert du fait divers comme d'un genre contre-culturel, est que celui-ci tombe luimême le 25 mars 1976. Il est kidnappé et porté disparu par le gouvernement militaire qui renversera un autre gouvernement péroniste, celui d'Isabel Perón.

Cette troisième partie, *Le procès ", se conclut par un épilogue dans lequel le journaliste élucide le massacre du dépotoir. Il déclare que les responsables «sont les signataires des décrets qui ont validé ces homicides: les généraux Aramburu et Ossorio Arana, les amiraux Rojas et Hartung, le brigadier Krause " (p. 194).

Contre toute apparence, Opération massacre est loin cependant d'être conclu. C'est un fait divers qui a pris une telle ampleur que le récit reste inachevé. Dans une nouvelle publication de la troisième édition, en 1971 , nous constatons que le récit continue après l'épilogue, dans un chapitre intitulé "Aramburu et le jugement historique ". Ce nouveau chapitre efface les marques subjectives des éditions antérieures et contient des indices paratextuels: le texte de ce chapitre apparaît en italique pour montrer qu'il n'appartient pas au corps textuel, mais plutôt qu'il découle de lui. Ce micro-texte devient très important dans notre hypothèse, car il sert de lien entre le texte initial de Walsh et les textes journalistiques de la gauche intellectuelle péroniste qui paraîtront plus tard, au commencement des annés soixante-dix. Nous voulons démontrer que ce micro-texte, *Aramburu et le jugement historique , préfigure un nouveau genre journalistique pour ainsi dire centré sur la mort.

\section{Le micro-texte: Opération massacre et les meurtres historiques}

L'originalité de cet agrégat est qu'il introduit le discours de la guérilla urbaine du point de vue de la gauche péroniste. L'épilogue laisse entendre qu'Aramburu deviendra la cible de l'opinion populaire et passera à l'histoire comme l'auteur du massacre du dépotoir du 10 juin 1956. Dans *Aramburu et le jugement historique ", nous retrouvons deux sujets reliés à la mort. Le premier 
concerne l'assassinat d'Aramburu, perçu comme un acte de justice du peuple péroniste après la mort du général Valle en 1956. Les circonstances de l'énonciation ont changé, les dates du récit en sont un premier indice (1956-1970). À l'époque de son interdiction, le péronisme subira une série de transformations idéologiques dont la plus remarquable sera la lecture marxiste qu'en feront les intellectuels des années soixante. De là, naîtra la guérilla urbaine péroniste de gauche.

L'instance énonciatrice, sujet de l'énonciation historique et de la chronique journalistique, exclut toute marque autobiographique. La figure de l'auteur empirique s'efface. L'auteur reste fidèle à son propos d'historien et proscrit tout ce qui est étranger au récit des événements. Personne ne parle ici. Par contre, si l'on reconstruit les circonstances historiques de cet événement, le *je * du chroniqueur des première, deuxième et troisièrne parties ("Les protagonistes", "Les faits " et *Le procès ") cède sa place à la troisième personne non référentielle. Dans * Aramburu et le jugement historique ", l'énonciateur raconte la genèse de l'exécution du général Aramburu depuis son enlèvement le 29 mai 1970, jusqu'à l'exécution elle-même par le commando *Montonero Valle ${ }^{13}$, le $1^{\text {er }}$ juin 1970 . Il est intéressant de noter ici que ce commando porte le nom du général Valle, et que *Montonero - sera le nom adopté par un groupe armé de guérilla urbaine qui comptera sur l'appui de la majorité des intellectuels du péronisme de gauche.

L'exécution d'Aramburu entraine des effets au niveau symbolique. Le jugement historique dont il est l'objet se présente aussi comme un échange de cadavres! Le cadavre d'Aramburu devient célèbre lors d'une dispute qui implique un autre cadavre légendaire, celui d'Evita Perón. Le commando . Montonero . avait exécuté Aramburu parce que l'armée n'avait pas satisfait leur

13 - Montonero serait d'origine portugaise: montoeira, "gros tas, agglomération *. Par contre, Rosenblat écrit que "montonera" est une création du Río de la Plata, typique du XIX' siècle et dont l'usage s'est généralisé dans toute l'Amérique hispanique comme le mot "guerrilla *. - Montonero. apparaît pour la première fois en référence à l'armée révolutionnaire d'Artigas au XIX' siècle et désigne donc une armée irrégulière. La *montonera ", d'autre part, a servi à désigner les guerres entre - gauchos. sous la direction de - caudillos *. Voir Diego Abad de Santillan, Diccionario de Argentinismos, Buenos Aires, Tipográfica, Editora Argentina, 1976. 
demande, le retour au pays du cadavre d'Evita Perón ${ }^{14}$. Lors du coup d'État de 1956, les militaires de la *Révolution libératrice . avaient caché le cadavre d'Evita hors du pays. Plus tard, les masses populaires péronistes revendiquèrent le retour au pays des restes d'Evita Perón. Son cadavre se transforma très vite en objet symbolique du péronisme interdit.

Le deuxième sujet d' *Aramburu et le jugement historique" est le renouvellement de la littérature nationale sur ces morts historiques. L'entrecroisement du politique et du littéraire vise à remettre en question ce qu'on entend par national. Ce texte suggère une définition de l'identité nationale qui inclut le péronisme. Les conflits à l'intérieur du champ littéraire sont témoins de la variété des prises de positions par rapport à cette nouvelle identité. À cette époque-là, Cortázar choisit l'exil volontaire, tandis que le groupe *Contorno" propose une révision du péronisme. Walsh, de son côté, désire incorporer le péronisme à la définition de l'identité nationale en combinant écriture et action politique.

Dans *Aramburu et le jugement historique", le traitement de la mort nous renvoie au passé et annonce l'avenir de ce mouvement péroniste de gauche. La littérature sert de point de référence. Le texte mentionne l'écrivain Ernesto Sábato et son roman Sobre bêroes y tumbas (1961) ${ }^{15}$ qui raconte, à partir de sources historiques, l'assassinat de Lavalle, autre général argentin du XIX siècle responsable de la mort de Dorrego. L'énonciateur n'hésite pas à faire le parallèle entre le général Aramburu et le général Lavalle et ainsi à offrir une nouvelle interprétation historique. La comparaison est basée sur la substitution suivante: dans *Aramburu et le jugement historique ", Aramburu est l'assassin de Valle; dans Sobre béroes y tumbas, Lavalle est l'assassin de Dorrego. Lavalle et Dorrego, tout comme Aramburu et Valle, symbolisent des factions politiques opposées. Dans le premier cas (Lavalle/

14 À partir de ces événements concernant le cadavre d'Evita Perón, Walsh écrit une nouvelle intitulée : Cette femme .

15 Ernesto Sábato, écrivain argentin dont la position idéologique illustre les transformations de certains groupes d'intellectuels par rapport au péronisme. Lors de sa première étape (El túnel et Sobre béroes $y$ tumbas), Sábato était anti-péroniste, mais s'engagea par la suite dans la lutte contre la répression militaire dés années soixante-dix. Dès les années quatre-vingt, il est devenu un des leaders de la défense des droits humains en Argentine. C'est à cette époque qu'il écrivit la préface de Nunca Más, recueil d'enquêtes sur les disparus. 
Dorrego), il s'agit des *unitarios * et des "federales* et, dans le deuxième cas (Valle / Aramburu), des "péronistes * et des *antipéronistes ". Au XIX siècle, comme à l'époque du récit, l'extrême virulence du conflit politique est une constante de la vie argentine. En invoquant ces assassinats historiques, cet ajout nous renvoie au texte initial, Opération massacre, et à l'assassinat politique des gens ordinaires du dépotoir. Il est intéressant de constater qu'à l'origine la littérature argentine est remplie de cadavres célèbres ou anonymes. Dans ce panthéon, la figure la plus puissante est celle du "caudillo* assassiné, Facundo Quiroga, protagoniste du récit fondateur de la littérature argentine, Facundo (1851) de Domingo Faustino Sarmiento. Barbara Crespo souligne à ce propos que, dans Opération massacre, on croit reconnaître le même ton que celui de Sarmiento au début de Facundo:

Spectre terrible de Facundo, je vais t'interpeller pour que, secouant la poussière ensanglantée qui couvre tes cendres, tu te lèves pour nous expliquer la vie secrète et les luttes internes qui déchirent les entrailles d'un noble peuple! ${ }^{16}$

\section{Opération massacre:}

Dépotoir sinistre de José León Suărez, abîmé de fossés inondés en hiver, infesté de grosses mouches bleues en été, rempli d'insectes morts et putréfiés, masse rongée de tôle et de ferraille, hérissée d'herbes rêches, dernier cloaque du monde, vois la charge qu'on t'apporte! ${ }^{17}$

Dans Opération massacre, le grand fantôme fondateur de la littérature argentine, l'ombre du "caudillo * Facundo ${ }^{18}$ continue à noircir l'imaginaire argentin qui a fait de l'homicide sanglant le trait caractéristique de l'identité nationale. N'oublions pas que la

16 Original espagnol: «;Sombra terrible de Facundo, voy a evocarte, para que, sacudiendo el ensangrentado polvo que cubre tus cenizas, te levantes a explicarnos la vida secreta y las convulsiones intérnas que desgarran las entrañas de un noble pueblo!s, Facundo, Madrid, Alianza Editorial, 1970, p. 17.

17 ¡ invierno, pestilente de moscas gordas y azules en verano, insultado de bichos muertos insepultos, corroído de latas y chatarra, velludo de pastos acerbos, último sumidero del mundo, mira la carga que te traen! :, p. 30-40.

18 Facundo: Civilización y Barbarie de Sarmiento (1851) est le récit fondateur de la littérature argentine. Il raconte la lutte politique de l'époque de la constitution de l'Argentine moderne et plus particulièrement l'assassinat de Facundo Quiroga, un personnage historique. 
40

première nouvelle nationale, le récit romantique d'Esteban Echeverría, s'intitule *L'abattoir * - .El matadero* - (1837) et que, par ce titre même, il évoque des morts sanglantes.

En somme, Opération massacre, en tant que récit sur les homicides politiques, nous renvoie aux origines de la littérature nationale. Cette tradition littéraire centrée sur des homicides politiques existe, depuis la deuxième moitié du XTX $\mathrm{X}^{\mathrm{e}}$ siècle, avec la tyrannie de Juan Manuel de Rosas, jusqu'aux années cinquante du XXe siècle, sous la régime de Juan Domingo Perón. Cette productivité textuelle nous amène à nous interroger sur le rapport entre ces deux textes et sur la séduction que peut présenter un texte artistique construit à partir de la répétition d'un événement sanglant. Opération massacre, comme récit, reprend la tradition de la série nécrophilique et, en tant que fait divers, dramatise l'homicide d'une façon spectaculaire.

\section{Les revues de la gauche péroniste. Du fait divers à la guerre populaire. Opération massacre dans la guerre populaire}

Et l'histoire est publiée: c'est une liasse de petites feuilles jaunes...

Feuilles éparses, les récits de Walsh rejoignent, au moins par leur forme et leur contenu, toute une série de narrations qui formaient alors une mémoire populaire des crimes politiques. Ils nous informent sur les motifs des assassinats qui alimentent l'imaginaire politique. La mémoire d'Opération massacre nous revient, trente-cinq ans après, comme un texte d'une puissance et d'une séduction telles que ce récit est devenu le texte fondateur d'une série d'autres textes qui s'élaborent eux aussi en marge du journalisme officiel. Opération massacre constitue le modèle de base des revues et des journaux de la gauche péroniste des années soixante-dix tels que El descamisado, Militancia, Causa Peronista, El Mundo, Ya et Hombre Nuevo. Le crime décrit dans Opération massacre est resté marqué dans la mẻmoire collective comme l'un des premiers assassinats anti-péronistes. Les homicides dont traitent toutes ces revues s'inscrivent à l'intérieur d'une lutte idéologique interne du péronisme dont le point de départ nous renvoie à Rodolfo Walsh, exemple parfait des transforma- 
tions que subit l'intellectuel engagé des années soixante et soixante-dix. En 1950, lors du premier mandat péroniste, Walsh était anti-péroniste, mais il se convertit progressivement à une nouvelle manifestation du péronisme dans les années soixantedix, un péronisme dit de gauche. Donc, l'affiliation politique de Walsh va de l'opposition au premier péronisme (1955), dans laquelle on retrouve aussi bien des gens de droite que de gauche, à la conversion à ce nouveau péronisme. Walsh, comme beaucoup d'intellectuels de sa génération, après la répression lancée par la "Révolution libératrice * contre le mouvement ouvrier, choisira de s'identifier à ce mouvement politique. Alors que Cortázar se joint aux intellectuels anti-péronistes et adopte les modèles esthétiques de la "narrativa del Boom *, Walsh choisit la chronique journalistique et un type d'écriture dénonciatrice. Ce type de journalisme ne vient pas du péronisme, mais se rapproche progressivement des groupes plus radicaux, particulièrement au journalisme du groupe "Montoneros *, adopté par la majorité des intellectuels à cette époque-là.

Dans Opération massacre, l'ajout *Aramburu et le jugement historique* est le premier récit qui traite de l'homicide politique dans le cadre d'une guerre populaire que les organisations de guérilla livreront entre 1973 et 1975 pendant le troisième gouvernement péroniste jusqu'à 1979. Les revues de gauche prolifèreront entre 1973 et 1976 . Peter Waldmann affirme que dans ces revues le traitement de la mort a connu sa glorification et sa mystification. L'hypothèse de Waldmann est que l'on retrouve dans ces revues et ces journaux, aussi bien dans les photos que dans le texte, un culte de la violence de type nazi ou stalinien: l'individu est une représentation de sa classe sociale, on ne tient pas compte de ses idées et de ses convictions personnelles. La plupart des cas de terrorisme se réduisent au type du simulacre terroriste, souligne Waldmann. Il analyse cette production culturelle centrée sur les assassinats politiques en termes de * sous-culture terroriste. Ces revues s'intéressent aux mouvements spontanés des secteurs sociaux défavorisés, aux grèves, aux occupations d'usines, aux mouvements de solidarité de la population avec les gens des bidonvilles ou avec les travailleurs syndiqués qui défient la bureaucratie syndicale accusée de corruption. Elles nomment les véritables acteurs des événements, notamment les travailleurs, les paysans, les femmes au foyer et les guérilleros. Ces revues sont d'une importance non négligable du point de vue documentaire, car c'est la première fois qu'un tel type de presse s'intéresse 
à ces secteurs sociaux, jusque-là négligés par les médias officiels $^{19}$. En plus, il faut souligner leur apport à la construction de l'identité péroniste de la classe ouvrière argentine. En effet, dans ces revues, on assiste à la répétition d'un événement du même genre: les récits d'assassinats politiques. Ces derniers sont liés, comme les ouvriers d'Opération massacre, au péronisme. Les victimes sont, elles aussi, élevées au statut de héros. Par exemple, on lira fréquemment dans ce type de revue: "Il a été tué parce qu'il était péroniste *

Chez l'essayiste Waldmann, la vérité scientifique tend à éliminer tout mystère. Par contre, le fait divers, au lieu d'évacuer le mystère, maintient cette ambiguïté entre l'intelligible et l'irrationnel. Tous ces récits de meurtres de petites gens devenus héros de la nation, de même que tous ces événements symboliques tels que le vol du cadavre d'Evita Perón, la mutilation du cadavre de Juan Perón, la décision du président péroniste Saul Menem de rapatrier d'Angleterre les cendres du cadavre de Juan Manuel de Rosas en octobre 1989 (pour ne mentionner que les derniers événements de la tradition péroniste) illustrent clairement la fonction sociale de certaines pratiques culturelles et politiques nourries par le fait divers ${ }^{20}$.

Comme Angel Rama, il faut considérer l'œuvre de Rodolfo Walsh par rapport à .l'émergence de sous-cultures dominées. à l'intérieur d'un champ intellectuel en conflit avec d'autres systèmes sémio-idéologiques. Rama, tout en suivant les études d'Antonio Gramsci sur la culture nationale, s'intéresse à l'œuvre de Walsh en tant que produit culturel d'une "sous-culture dominée" pénétrant la culture hégémonique. De cette façon, Opération massacre, tout comme les drames policiers du XIX ${ }^{e}$ siècle, dont les manifestations les plus éloquentes sont les feuilletons d'Eduardo Podestá, s'inscrirait dans la tradition du feuilleton argentin. La popularité de ces formes culturelles est reliée aux sentiments de ceux qui consomment ces histoires de mauvais "gauchos ", dont le Juan Moreira d'Eduardo Podestá est un bon

19 Peter Waldmann, Anomia social y violencia., Argentina boy, Alain Rouquié (comp.), Mexico, 1982, p. 206-249.

20 Tomás Eloy Martínez affirme que cette cérémonie faisait partie d'une chaîne de rapatriements qui incluaient aussi d'autres cadavres comme celui de Jorge Luis Borges et de l'éphémère président péroniste Héctor J. Cámpora. Voir Tomás Eloy Martínez, "La necrofilia argentina ", Todo es Historia, 1992. 
exemple; ce type de récit est écrit pour satisfaire les demandes de justice des couches populaires confrontées à un appareil policier et judiciaire inefficace ${ }^{21}$. Donc, en surenchérissant sur la proposition de Peter Waldmann de "sous-culture terroriste * et sur celle d'Angel Rama de "sous-cultures dominées * nous voulons souligner le rapport que la production de Walsh comme celle des revues politiques entretient avec le fait divers en tant que pratique sous-culturelle. Cette production à caractère politique et journalistique, tout en prenant des éléments distinctifs du fait divers, renforce d'autant sa nature sous-culturelle ou contreculturelle.

Le traitement de l'assassinat politique dans Opération massacre et dans les revues de gauche démontre un lien étroit entre le péronisme et le fait divers. En tant que récit, le fait divers occupe une zone dangereuse: il fait communiquer l'interdit et la soumission. Avec "l'anonymat du héros l'infamie touche à l'éternité ", nous rappelle Foucault ${ }^{22}$. Dans certains milieux argentins, particulièrement dans les milieux militaires, le péronisme représente l'interdit, il constitue un phénomène irrationnel, une sorte de culte religieux. L'épigraphe de John W. Cooke au début de notre texte l'illustre assez bien. Dans les couches populaires, un certain mysticisme entoure le péronisme, à tel point que l'étude de la conscience collective doit en tenir compte. Il était courant, à cette époque-là, et cela reste encore vrai aujourd'hui, de retrouver dans les maisons des travailleurs des photos d'Evita et de Perón placées avec des photos de saints sur le petit oratoire familial. L'évolution politique de l'Argentine contemporaine est due, dans une large mesure, aux transformations du péronisme. Le régime péroniste a toujours été très proche des milieux populaires et intellectuels de la génération des années soixante-dix, c'est-à-dire de ceux qui s'étaient tournés en masse vers un péronisme marxiste. Cet *air du temps * issu de la lecture marxiste du péronisme par les intellectuels des annés soixante reste encore très énigmatique. José Pablo Feinman, dans son roman La astucia de la razón (1990), s'adresse à cette génération des intellectuels convertis au péronisme. Sa réflexion prend comme référence la position péroniste de John W. Cooke, pour qui les convictions péronistes de la classe

21 Angel Rama, op. cit.

22 Michel Foucault, Moi Pierre Rivière, ayant égorgé ma mère, ma scuur et mon frère..., Paris, Gallimard/Julliard, 1973. 
ouvrière étaient ressenties par l'État bourgeois comme un fait divers, c'est-à-dire, *maudit * et * irrationnel *. C'est sur ce fond de conflit interne au péronisme des années soixante-dix que les revues gauchistes, suivant le modèle établit par Walsh, communiquent à l'histoire ces meurtres sacrificiels et glorieux. On pourrait donc conclure que ces intellectuels nés pendant la période d'interdiction du péronisme, en principe anti-péronistes comme Walsh, deviennent péronistes plus tard, parce que, comme l'affirmait John W. Cooke, l'idéologue de cette lecture gauchiste du péronisme, être pour la classe ouvrière, c'est accepter la nature populiste du péronisme. En outre, une fois encore, l'irrationalité pénètre la raison. En ce sens, Opération massacre devient un symbole qui s'ajoute aux autres figures du panthéon, morts historiques déjà mentionnés, de l'imaginaire collectif.

Les morts d'Opération massacre renforcent le désir de justice de la classe ouvrière péroniste. Au niveau de l'imaginaire collectif, ce désir de justice rejette inconsciemment l'irréversibilité de ces morts afin de pouvoir faire face à l'interdiction et à la répression politique. Ce rejet au niveau symbolique transforme ces morts en éléments constitutifs de l'identité nationale argentine ${ }^{23}$. L'assassinat de Facundo Quiroga, ainsi que les morts sacrificielles d'Opération massacre et celles des revues de gauche, ont eu lieu à des moments de crise, de disparition ou d'interdiction. L'interdiction et la répression du péronisme à deux reprises (1956 et 1976) génèrent ces éléments de l'identité nationale qui, paradoxalement, sont incorporés dans l'imaginaire pour ainsi reconstruire le vide laissé par l'interdiction. L'irrationalité des homicides contre les péronistes, supprimée au niveau de la conscience, est ainsi rejetée au niveau pulsionnel. Nous avons tenté, dans cet article, de démontrer que le texte de Walsh, Opération massacre, est un modèle de pratique narrative populaire contre-culturelle qui permet d'exercer une action politique par le truchement d'un fait divers dans un monde de répression.

23 À ce propos, consulter l'étude particulièrement intéressante d'Enriqueta Ribé, - Barranca Yaco, literatura, acontecimiento e historia *, Revista de Letras, $\mathrm{n}^{\circ} 2,1990$, p. 38-42. 